\title{
| HEART RATE VARIABILITY AND SKIN CONDUCTANCE BIOFEEDBACK: A TRIPLE-BLIND RANDOMIZED CONTROLLED STUDY
}

Steven Raaijmakers, William Steel, Maartje de Goede, Nelleke van Wouwe, Jan van Erp and Anne-Marie Brouwer TNO Soesterberg, the Netherlands

anne-marie.brouwer@tno.nl

\section{2 innovation for life}

\section{INTRODUCTION}

Low heart rate variability (HRV) and high skin conductance level (SCL) are associated with stress (suppressed parasympathetic and activated sympathetic autonomous nervous system). Low HRV has been linked to (psycho)pathological illnesses and lower performance on cognitive tasks.

When no stress response is required, increasing ones HRV and decreasing ones SCL may be beneficial.

This may be achieved by biofeedback: providing an individual with continuous information about own physiological signals with the aim of learning to modulate them. This should lead to improvement in mental or physical condition.

There is a lack of properly controlled biofeedback research - none seems to exist for HRV.

Current research question:

Does an existing biofeedback game targeting HRV and SCL indeed lead to increased HRV and

decreased SCL in rest? Does this have positive

effects on several cognitive, affective and EEG alpha asymmetry ${ }^{1}$ measures?

\section{METHOD}

Participants

28 healthy male students, $19-27$ years.

Task

Playing 'Virtual Remote Dome Controller' in which vehicles and equipment should be controlled through either HRV, SCL or both. For HRV, visuals on the screen encouraged participants to breath at 0.1 $\mathrm{Hz}$ (which increases HRV). Participants were told to find out themselves how to control the game.

Design and effect measures

One practice session and six experimental sessions on separate days.

Pre- and post effect measures on day 1 and day 7 :

- Physiological: HRV, SCL ${ }^{2}$, EEG alpha asymmetry

- Cognitive: n-back and mental rotation task (reaction time and error percentage)

- Affective: PANAS questionnaire (negative and positive affect)

Two groups of participants:

- Biofeedback $(n=16)$ : game responded to physiology

- Control ( $n=12)$ : physiology was not used participants received simulated gaming feedback that modeled learning as in biofeedback group

Triple-blind: participants and experimenters did not know group assignment until the end of the analysis.

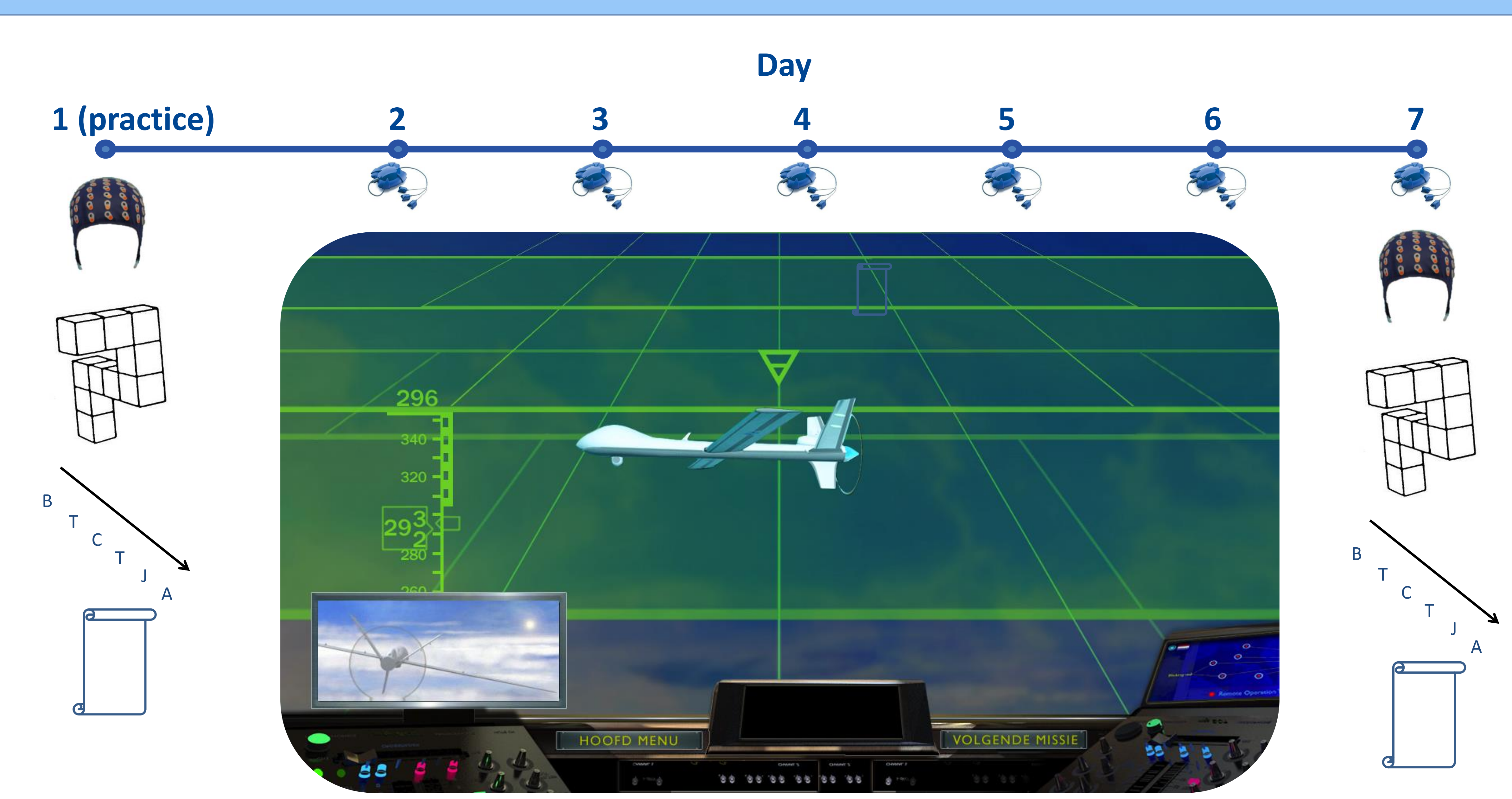

\section{RESULTS}

Repeated measures ANOVAs with group as between and time of measurement as within-subject variable for all effect measures.

- Effect of time of measurement on n-back task (reaction time and error percentage) consistent with learning.

- No effect of group and no interaction between group and time of measurement on any of the effect measures.

\section{DISCUSSION}

We did not find any effect of real biofeedback treatment versus a triple-blind control treatment.

Possible reasons include:

- More training sessions needed

- Non-optimal training paradigm

- Only helpful for a special (stressed) population

Biofeedback in general has not been disproven by this study. However, there seem to be no studies that show benefits of biofeedback (targeted at the autonomic nervous system) over and above nonspecific treatment effects.

${ }^{1} \mathrm{An}$ increase in right frontal EEG activity (as measured as a decrease in alpha) would be indicative of a stronger modulation of cardiac activity.

2 Data only available from 9 biofeedback and 7 control participants.

\section{ACKNOWLEDGEMENT}

This work is supported by the BrainGain Smart Mix Programme of the Netherlands Ministry of Economic Affairs and the Netherlands Ministry of Education, Culture and Science. 